\title{
PLATELET-RICH FIBRIN TREATMENT OF MEDICATION-RELATED OSTEONECROSIS OF THE JAW FOLLOWED UP FOR TWO YEARS
}

\author{
Meri Hristamyan $^{1^{*}}$ and Veselka Hristamyan ${ }^{2}$ \\ ${ }^{1}$ Department of Epidemiology and Disaster Medicine, Faculty of Public Health, Medical University, \\ Plovdiv, Bulgaria, ${ }^{2}$ Clinic of Maxillofacial Surgery, St George University Hospital, Plovdiv, Bulgaria
}

The use of platelet-rich fibrin (PRF) in surgery represents a new alternative method of treatment of Medication-related osteonecrosis of the jaws. This report presents a case of a patient undergoing PRF treatment. The patient is a 76-year-old male who was admitted for treatment in 2018 at the Clinic for Maxillofacial Surgery with osteonecrosis of the lower jaw on the right side. In 2012, he was diagnosed with prostate cancer and underwent bilateral orchiectomy followed by a therapy with Denosumab (trade names Prolia and Xgeva), a monoclonal antibody that works as receptor activator of nuclear factor kappa-B ligand (RANKL) inhibitor. The patient was diagnosed with medication-related osteonecrosis of the mandible jaw in 2017 after tooth extraction. The treatment was performed at the Clinic of Maxillofacial Surgery of St. George University Hospital, Plovdiv, Bulgaria. When initial antibiotic therapy failed, surgical therapy was undertaken using PRF. The patient was followed-up for two years and no osteonecrotic lesion was detected recurring in the intervention area. Biomed Rev 2020; 31: 161-163

Keywords: platelet-rich fibrin, jaw osteonecrosis, maxillofacial surgery

\section{INTRODUCTION}

The use of platelet-rich fibrin (PRF) in surgery is a new alternative method of treatment of Medication-related osteonecrosis of the jaws (MRONJ) (1). In 2006 Dohan et al (2) develop a PRF production protocol in an attempt to accumulate platelets and release cytokines in a fibrin clot. Platelets and leukocytes are an important part of this biomaterial, but the fibrin matrix that supports them is very useful in compiling responsible determinants for the therapeutic potential of PRF (2).

The use of a PRF membrane in addition to wound healing and periodontal regeneration has shown promising results. It is successfully used for the correction of bone defects in periodontics, oral and maxillofacial surgery and implant dentistry. However, most PRF studies show only short-term results. More controlled clinical trials with long-term results are needed to gain a deeper understanding of the effectiveness and reliability of this biomaterial in the long term and to

*Correspindence to: Dr Meri Hristamyan, Department of Epidemiology and Disaster Medicine, Faculty of Public Health, Medical University of Plovdiv, 15A Vassil Aprilov Blvd, BG-4000 Plovdiv, Bulgaria. E-mail: mary_hr@abv.bg Tel: +359 88 3399364 
optimize its use in day-to-day procedures (3)

\section{CASE REPORT}

The patient is a 76-year-old male, who in 2018 received treatment at the Clinic of Maxillofacial Surgery of St. George University Hospital, Plovdiv, Bulgaria, with a diagnosis of medication-related osteonecrosis of the right side of the mandible (Fig. 1).

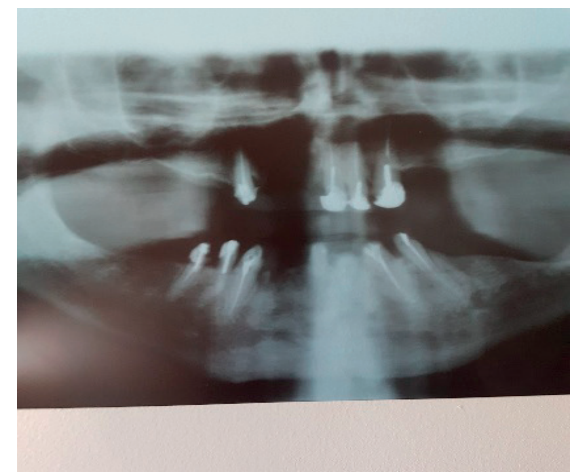

Figure 1. Orthopantomography on admission to the clinic.

In 2012, the patient was diagnosed with prostate cancer and underwent bilateral orchiectomy. In 2013, scintigraphy (Fig. 2) revealed metastases in the bones and following the recommendation of the oncohematologist, the patient was treated with Denosumab (trade names Prolia and Xgeva), a monoclonal antibody that works as receptor activator of nuclear factor kappa-B ligand (RANKL) inhibitor, classified as a "bone-modifying agent" linked to cases of MAONJ (4).

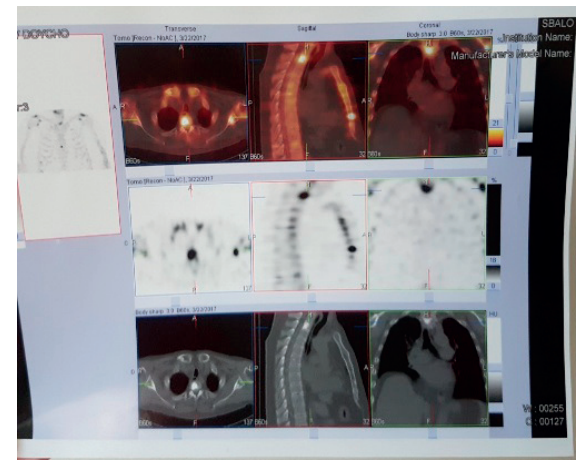

Figure 2. Scintigraphy of bone metastases.

Medication-related osteonecrosis of the mandible was diagnosed in 2017, after dental extraction in a dental office. The patient was referred for treatment at the Clinic of Maxillofacial Surgery of St. George University Hospital, Plovdiv, Bulgaria, where initially antibiotic therapy was undertaken. This conservative therapy led to no improvement.

As a next step, surgical treatment was performed using PRF. The standard operating protocol included: a mucoperiosteal flap repaired under local anesthesia; excision of the osteonecrotic lesion; hemostasis; PRF placement (Fig.3); and suture of the wound.

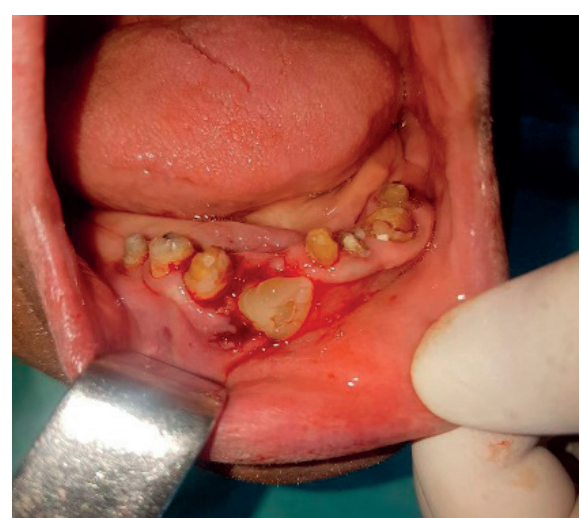

Figure 3. PRF placement.

In the postoperative follow-up period, the patient's condition was monitored for two years and no osteonecrotic lesion was detected in this area (Fig. 4, 5).

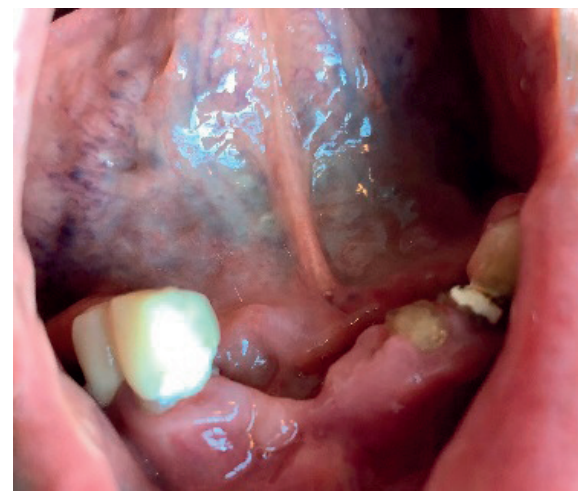

Figure 4. Follow-up one year after surgery.

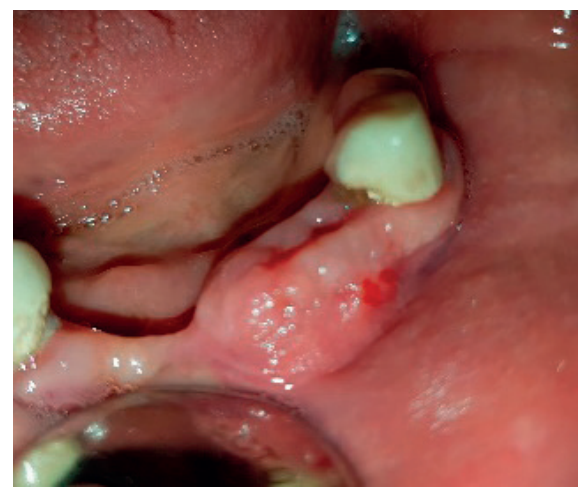

Figure. 5. Follow-up two years after surgery 


\section{DISCUSSION}

Surgical treatment remains one of the most reliable methods in the treatment of MAONJ, but it also needs to be updated and take advantage of advances in research and technologies that provide the opportunity for relatively easy and affordable use of new growth factors in maxillofacial surgery (3). One of these achievements is the PRF membrane, which can be obtained in an easy and accessible manner on an outpatient basis (5).

The use of PRF in surgery represents a new alternative method for the treatment of MAONJ (6). Dohan et al (2) develop a PRF production protocol for attempting to accumulate platelets and release cytokines in a fibrin clot. Platelets and leukocytes are an important part of this biomaterial, but the fibrin matrix that supports them is very useful in compiling responsible determinants for the therapeutic potential of PRF (7).

The use of a PRF membrane in addition to wound healing and periodontal regeneration has shown promising results (3). It is successfully used for the correction of bone defects in periodontics, oral and maxillofacial surgery and implant dentistry. However, most PRF studies show only short-term results. More controlled long-term clinical trials are needed to gain a deeper understanding of the effectiveness and reliability of this biomaterial long term and to optimize its use in daily procedures (8).

\section{CONCLUSION}

Compared to conservative treatment, PRF treatment shows much better overall long-term results with computer tomography follow-up at month 12 . PRF treatment is characterized by a significantly higher percentage of patients with high bone shadow intensity, whereas conservative treatment is dominated by a low bone shadow intensity (4).

Comparing the two types of treatment - conservative and alternative, in terms of homogeneity and outline, PRF patients have significantly better results at the $12^{\text {th }}$-month mark and lasting results 24 months after the intervention. PRF treatment is dominated by homogeneity and sequestration, whereas in conservative treatment, the rate of homogeneity and sequestration is lower and the presence of heterogeneity is also found (9). In patients with PRF treatment, sharp and continuous outlines are predominant, whereas, in conservative patients, blurred and interrupted outlines are more common. $\mathrm{PRF}$ is a second-generation platelet concentrate obtained naturally without the addition of thrombin and anticoagulant and has a natural fibrin framework that can protect the growth factors from proteolysis.

In the reported case, the condition of the patient was monitored for two years and no recurring osteonecrotic lesion was detected in the area treated with PRF.

\section{CONFLICT OF INTEREST}

None.

\section{ACKNOWLEDGMENTS}

The paper was supported by the Ministry of Education and Science, Republic of Bulgaria (National program "Young scientists and postdoctoral students").

\section{REFERENCES}

1. American Association of Oral and Maxillofacial Surgeons. https://www.aaoms.org/

2. Dohan DM, Choukroun J, Diss A, Dohan SL, Dohan AJ, Mouhyi J, Gogly B. Platelet-rich fibrin (PRF): a secondgeneration platelet concentrate. Part II: platelet-related biologic features. Oral Surg Oral Med Oral Pathol Oral Radiol Endod 2006;101(3):e45-50. doi: 10.1016/j. tripleo.2005.07.009.

3. Tsolov R. Treatment of bisphosphonate necrosis of the jaws with the help of platelet-rich fibrin. Med Magazine 2019; 61: 120-123.

4. Tsolov R, Firkova E, Yordanov G. Comparative analysis of the healing process after conservative and surgical treatment of Medicamentally Induced Osteonecrosis of the Jaws. Bul Med J 2019; 2: 21-26.

5. Bartl R, Frisch B, von Tresckow E, Bartl C. Bisphosphonates in Medical Practice Actions, Side Effects, Indications, Strategies. Berlin/New York: Springer. 2007.

6. Chandran P, Sivadas A. Platelet-rich fibrin: Its role in periodontal regeneration. Saudi J Dent Res 2014;5:117122. doi: 10.1016/j.ksujds.2013.09.001.

7. Anitua E. Plasma rich in growth factors: preliminary results of use in the preparation of future sites for implants. Int J Oral Maxillofac Implants 1999;14(4):529-35.

8. Asaka T, Ohga N, Yamazaki Y, Sato J, Satoh C, Kitagawa Y. Platelet-rich fibrin may reduce the risk of delayed recovery in tooth-extracted patients undergoing oral bisphosphonate therapy: a trial study. Clin Oral Invest 2017;21(7):2165-2172. doi: 10.1007/s00784-016-2004-z.

9. Tsolov R, Firkova E, Yordanov G. Bisphosphonate induced osteonecrosis of the jaws - X-ray results 6 months after ureatment. Contemp Med 2019; 1:13-30. 